\title{
Hipertensión arterial y tratamiento del cáncer
}

\author{
Dr. Horacio Vázquez Nosiglia
}

\section{Resumen}

La hipertensión arterial (HTA) tiene alta prevalencia y es la comorbilidad más frecuente al momento del diagnóstico de cáncer. La nueva aparición o el agravamiento de la HTA durante el tratamiento oncológico es también común, sobre todo durante los períodos de quimioterapia. Si bien varias drogas utilizadas en quimioterapia tienen potencial pro hipertensivo, los fármacos inhibidores de la angiogénesis tienen un efecto de clase sobre la presión arterial (PA) y son los que plantean mayores problemas al médico. Los tratamientos de sostén también pueden incrementar la PA, sobre todo la eritropoyetina, los antiinflamatorios no esteroideos y los corticoesteroides. No existe una guía específica basada en evidencia para el manejo de la HTA durante el tratamiento del cáncer, por tanto se revisan las recomendaciones de expertos para enfrentar este problema frecuente.

Palabras clave: HIPERTENSIÓN

NEOPLASIAS

QUIMIOTERAPIA

INHIBIDORES DE LA ANGIOGÉNESIS

\section{Hypertension and cancer treatment}

\section{Summary}

Hypertension is highly prevalent and the most frequent comorbidity at the initial diagnosis of cancer. New onset or worsening hypertension is also common during cancer treatment, in particular during chemotherapy periods. Several drugs used in chemotherapy could have an hypertensive effect, for instance angiogenesis inhibitors have a class effect on blood pressure and frequently clinicians confront this problem. Adjuvant drugs in cancer treatment could also increase blood pressure, especially erythropoietin, non-steroidal anti-inflammatory drugs and corticosteroids. There are not specific evidence based guidelines for the management of hypertension during cancer treatment, for that reason we review expert suggestions to deal with this frequent issue.

Key words: $\quad$ HYPERTENSION

NEOPLASMS

CHEMOTHERAPY

ANGIOGENESIS INHIBITORS

\section{Convergencia epidemiológica}

La prevalencia promedio de hipertensión arterial (HTA) en la población adulta (mayor de 25 años) de las Américas ha sido estimada en 35\%, según un reporte de la Organización Mundial de la Salud del año $2013^{(1)}$. Esta prevalencia es creciente con la edad, por lo que se encuentra presente en la mayoría de las personas que tienen más de 60 años. La prevalencia de cáncer también aumenta con la edad y comparte varios factores de riesgo con la enfermedad cardiovascular (tabaco, dieta inadecuada, obesidad, sedentarismo). La HTA constituye la comorbilidad más frecuente entre los pacientes con diagnóstico inicial de cán- cer, con una prevalencia que se ha estimado próxima a $40 \%$ o mayor en esa población ${ }^{(2-4)}$. Asimismo, estudios epidemiológicos recientes ajustados por edad y otras variables han encontrado un riesgo aumentado de cáncer y de mortalidad por esta causa entre los pacientes hipertensos ${ }^{(5,6)}$.

La aparición de nuevos casos de HTA o el agravamiento de la misma en quienes ya la padecen es muy común durante el tratamiento del cáncer. En un estudio retrospectivo de más de 25.000 pacientes con varios tipos de cáncer, que registraron una presión arterial (PA) normal al momento del diagnóstico (independientemente de sus anteceden-

MP Medicina Personalizada, Montevideo,Uruguay.

Este artículo integrará el Manual de Cardio-Oncología de la Sociedad Sudamericana de Cardiología, coordinado por el Dr. Enrique Ruiz Mori (Perú), y se publica con autorización como un adelanto del mismo.

Recibido Feb 27, 2018; Mar 6, 2018. 
Tabla 1. Drogas antineoplásicas de uso extendido que son causa frecuente de hipertensión arterial.

$$
\begin{aligned}
& \text { Drogas alquilantes } \\
& \hline \text { - Cisplatino } \\
& \hline \text { Drogas inhibidoras de angiogénesis } \\
& \hline \text { Anticuerpos monoclonales } \\
& \text { - Bevacizumab } \\
& \text { Inhibidores de tirosina quinasa } \\
& \text { - Sorafenib } \\
& \text { - Sunitinib } \\
& \text { - Pazopanib } \\
& \text { - Vandetanib }
\end{aligned}
$$

tes), casi un tercio desarrolló algún grado de HTA durante el tratamiento oncológico (32,16 casos por 100 personas/año)(7). La mayoría desarrolló hipertensión moderada, pero 12,36 por 100 personas/año tuvieron cifras de PA sistólica entre 160 y $180 \mathrm{mmHg}$ y 2,79 por 100 personas/año tuvieron cifras de PA sistólica mayores de 180. Para cualquier grado de severidad de HTA, ella fue considerablemente mayor durante los períodos de exposición a quimioterapia, aunque debe mencionarse que el monitoreo también fue más intenso durante dichos períodos.

La incidencia de HTA es diferente según el tipo de tumor, pues es menor en melanoma, cáncer de mama y partes blandas, mientras que es mayor en cáncer renal, pulmonar, de ovario, próstata, colorrectal o gástrico. Los niveles más severos de HTA (PA sistólica $\geq 180 \mathrm{mmHg}$ ) se observaron con mayor frecuencia en pacientes con cáncer renal durante períodos de quimioterapia (19,54 por 100 personas/año), lo cual probablemente esté en relación con la disminución de la función renal (con frecuencia nefrectomía previa) y al tipo de drogas utiliza$\operatorname{das}^{(7)}$.

En el paciente oncológico el número y la calidad de las comorbilidades juegan un papel de primer orden en la calidad de vida y el pronóstico de la enfer$\operatorname{medad}^{(2,3)}$. En el pasado la HTA se consideraba poco relevante en una persona con una expectativa de vida comprometida por el cáncer. Sin embargo, ese concepto ha cambiado pues actualmente muchos pacientes tienen una sobrevida prolongada y además la propia HTA puede ser causada o agravada por el tratamiento oncológico, lo que se convierte en una limitante para la continuidad y eficacia del mismo.
En relación con la potencial cardiotoxicidad del tratamiento quimioterápico, la HTA ha sido identificada como factor de riesgo y también como factor causal de la misma. Por ejemplo, durante la terapia con antracíclicos en adultos con linfopatía tumoral, el antecedente de HTA implicó un incremento de riesgo de insuficiencia cardíaca de 1,58 (95\% IC, $1,28$ a 1,95$)^{(8)}$. Al mismo tiempo el desarrollo de HTA ha sido propuesto como uno de los mecanismos causales de disfunción ventricular izquierda entre los pacientes tratados con agentes inhibidores de la angiogénesis ${ }^{(9,10)}$.

\section{Quimioterapia e hipertensión arterial}

Varios agentes utilizados en quimioterapia tienen potencial pro hipertensivo. Desde hace más de 20 años las drogas alquilantes, principalmente el cisplatino, que tiene potencial nefrotóxico, y más raramente la ciclofosfamida, han sido relacionadas a una mayor prevalencia de HTA durante y después del tratamiento oncológico. Sin embargo, pese a que son extensamente utilizadas en varios tipos de cáncer, existen escasos reportes sobre la verdadera incidencia e importancia de la hipertensión asociada a su uso(4,11,12).

La introducción y uso creciente de las drogas inhibidoras de la angiogénesis (sinónimo de antiangiogénicas o inhibidoras de la vía del factor de crecimiento endotelial vascular [VEGF, por su sigla en inglés]) ha resaltado la importancia de la HTA durante el tratamiento del cáncer, pues como grupo son capaces de inducirla con una frecuencia que ha sido estimada variablemente entre el $17 \%$ y el $80 \%$ de los casos en diferentes estu$\operatorname{dios}^{(4,10,12,13)}$ (tabla 1).

\section{Drogas inhibidoras de la angiogénesis (IA) e hipertensión arterial}

El desarrollo de vascularización en los tumores sólidos es un paso limitante para su crecimiento y diseminación. Las células tumorales producen sustancias activadoras de la angiogénesis o pro angiogénicas, pues la neovascularización es imprescindible para el desarrollo de tumores mayores de 2 o $3 \mathrm{~mm}$. El VEGF está sobreexpresado en la mayoría de los cánceres sólidos, por lo que la inhibición de esta vía de señalización (signalling pathway) pro angiogénica se ha convertido en un objetivo preciso para suprimir el crecimiento tumoral ${ }^{(14)}$. El VEGF no solamente estimula la angiogénesis sino que es un factor de sobrevivencia del endotelio vascular y promueve un fenotipo de vasos sanguíneos anormales dentro de los tumores ${ }^{(15)}$. 
El mecanismo por el que estas drogas producen HTA no está completamente aclarado y probablemente sea multifactorial. La vía de señales del VEGF también está presente en el endotelio vascular normal jugando un papel fisiológico en su funcionamiento y en la síntesis de óxido nítrico, por lo que su bloqueo compromete la vasodilatación. Además de la disminución del óxido nítrico se han propuesto otros mecanismos como incremento de endotelina 1 , rarefacción microvascular, activación del sistema renina angiotensina, estrés oxidativo, daño glomerular renal y aumento de la rigidez arterial de los grandes vasos. Es frecuente que, junto con la HTA, las drogas IA induzcan algún grado de proteinuria, aunque es raro que por sí solas afecten sostenidamente la función renal(16).

Lo cierto es que el mecanismo de acción pro hipertensivo es un efecto de clase y está directamente relacionado a su acción antitumoral, por lo que el incremento de la PA, paradójicamente, puede ser un marcador de la efectividad del tratamiento oncológico. Ha sido documentado que los pacientes que desarrollan HTA tienen mejor respuesta al tratamiento con estas drogas y mayor sobrevida(17,18). En una serie retrospectiva de casi 5.000 pacientes tratados con sunitinib para carcinoma de células renales en etapa metastásica, los que desarrollaron HTA tuvieron una sobrevida promedio cuatro veces mayor (30,9 meses contra 7,2 meses) ${ }^{(19)}$. Afortunadamente el control de la PA con drogas antihipertensivas no parece afectar la efectividad del tratamiento oncológico.

\section{Modalidades de uso de las drogas inhibidoras de la angiogénesis}

El lugar principal de los agentes IA se encuentra en la etapa metastásica del cáncer, pero la respuesta no es igual en todos los tipos de tumores ${ }^{(14)}$.

Hay dos tipos de fármacos en amplio uso clínico que están apuntados a esta vía y ambos tienen efecto pro hipertensivo. El primero consiste en anticuerpos monoclonales dirigidos al VEGF, del cual la droga tipo es el bevacizumab (Avastin ${ }^{\circledR}$ ). Esta droga se administra por vía endovenosa en planes combinados con drogas citotóxicas, que pueden potenciar el efecto hipertensivo o generar efectos cardiotóxicos asociados ${ }^{(14)}$. El efecto pro hipertensivo de bevacizumab es dosisdependiente $\mathrm{y}$, debido a que tiene una vida media de unos 20 días, es prolongado luego de su administración endovenosa $^{(20)}$.

El segundo grupo de drogas apuntadas a esta vía son los inhibidores de tirosina quinasa, que actúan interfiriendo la unión de VEGF con su receptor. Las drogas de mayor uso en este grupo son sorafenib, sunitinib, pazopanib y vandetanib, aunque hay otras de más reciente desarrollo. En general se utilizan como monodroga de administración oral en planes de uso prolongado, por lo que aumenta el tiempo de exposición al efecto pro hipertensivo(14). También han sido asociadas a efecto cardiotóxico directo, que se ve potenciado por la elevación de la $\mathrm{PA}^{(9,14,21)}$.

\section{Magnitud del efecto pro hipertensivo de las drogas inhibidoras de la angiogénesis}

La HTA inducida por agentes IA se presenta con mayor frecuencia al inicio del tratamiento, típicamente durante las primeras cuatro semanas, aunque algunos casos pueden presentarse más tardíamente dentro del primer año del mismo. La mayoría de los pacientes desarrollan una HTA leve o moderada y la situación es manejable con la medicación antihipertensiva habitual. Sin embargo, algunos de ellos pueden sufrir un incremento dramático y a veces impredecible de la PA en los primeros días de comenzado el tratamiento ${ }^{(22)}$.

Cuanto más potente la inhibición de la vía de señalización de VEGF, mayor el potencial hipertensivo, por lo que el mismo es más frecuente y severo con las drogas de introducción más reciente (axitinib, regorafenib y lenvatinib)(18).

Con bevacizumab, la incidencia de cualquier grado de HTA en un metaanálisis con más de 12.000 pacientes fue de $23,6 \%$ (PA superior a 150/100 o incremento $>20 \mathrm{~mm}$ de PA diastólica en individuos previamente normotensos), en tanto 7,9\% desarrolló HTA grave ${ }^{(23)}$.

Con inhibidores de tirosina quinasa, en un registro de más de 1.000 pacientes tratados con sunitinib, sorafenib o pazopanib, se identificó un efecto hipertensivo inducido por el tratamiento en la mitad de los mismos (combinación de nuevo diagnóstico de HTA o agravación de HTA preexistente), lo que coincide con reportes previos en series más pequeñas. En este registro el incremento absoluto promedio de la PA fue $21 \mathrm{mmHg}$ (sistólica)/15 mmHg (diastólica), tanto en pacientes con HTA preexistente o sin ella ${ }^{(24)}$. Dos trabajos que realizaron registros de monitoreo ambulatorio de PA durante los primeros días de tratamiento con sorafenib o sunitinib mostraron incremento promedio de PA de similar o mayor magnitud que las referidas $^{(25,26)}$.

\section{Tratamientos de sostén que pueden inducir hipertensión arterial}

Numerosas drogas ampliamente utilizadas en la práctica médica pueden inducir HTA o incrementar 
las cifras presoras en pacientes que ya reciben tratamiento para la misma. Estas drogas son frecuentemente prescriptas en apoyo del paciente tratado por cáncer y pueden potenciar el efecto pro hipertensivo inducido por la quimioterapia ${ }^{(13)}$.

Los antiinflamatorios no esteroideos (AINE), la eritropoyetina y los corticoesteroides son los que con mayor frecuencia plantean este problema. Sin embargo, debe realizarse una detallada encuesta sobre cuáles de los medicamentos indicados pueden inducir HTA, incluyendo tratamientos hormonales (estrógenos, progestágenos), agentes inmunosupresores (ciclosporina A, tacrolimus, sirolimus), aminas simpático-miméticas (contenidas en descongestionantes nasales o antigripales), numerosos psicofármacos como las anfetaminas, el modafinilo, antipsicóticos (clozapina) y antidepresivos (inhibidores de la monoaminooxidasa, tricíclicos y algunos inhibidores selectivos de la recaptación de serotonina). No debe olvidarse que el consumo excesivo de alcohol, cafeína y algunas drogas de abuso (cocaína) también inducen $\mathrm{HTA}^{(27)}$.

\section{Eritropoyetina}

La anemia acompaña con frecuencia al paciente que recibe tratamiento por cáncer y la eritropoyetina es una hormona prescripta en algunos de ellos para estimular la formación de glóbulos rojos en la médula ósea. Han sido postulados varios mecanismos para explicar el efecto pro hipertensivo de la eritropoyetina, pero lo cierto es que alrededor de un tercio de los pacientes que reciben eritropoyetina recombinante muestran incremento de las resistencias vasculares periféricas y algún grado de elevación de la PA. El efecto hipertensivo puede manifestarse luego de las dos semanas de comenzar el tratamiento y la HTA no controlada puede ser motivo de su suspensión hasta en 15\% de los casos. Estos pacientes, por lo general, responden bien con manejo del volumen y con agentes antihipertensivos de cualquier clase (13,27). $^{2}$

\section{Antiinflamatorios no esteroideos}

Los AINE son utilizados en el manejo del dolor de múltiples causas, por lo que se indican regularmente a pacientes con cáncer.

Los AINE inducen HTA a través de inhibición de ciclo-oxigenasa (COX), lo que reduce la síntesis de prostaglandinas e induce vasoconstricción renal y retención hidrosalina. La intensidad del efecto pro hipertensivo puede variar entre las diferentes drogas. Se ha estimado que los normotensos que reciben regularmente un AINE duplican la posibilidad de desarrollar HTA, aunque el efecto es más no- table en los de mayor edad o en los portadores de algún grado de disfunción renal(27).

El consumo de AINE puede desestabilizar el control de la PA en hipertensos tratados con inhibidores de enzima conversora de angiotensina (IECA), antagonistas de angiontensina II (ARA II), betabloqueantes y diuréticos, dado que el efecto vasodilatador renal de las prostaglandinas es importante en el mecanismo de acción de estas drogas. En cambio, no se ha demostrado interferencia con las drogas calcioantagonistas o los bloqueadores adrenérgicos a nivel central.

\section{Corticoesteroides}

Los corticoesteroides tienen diversas aplicaciones en apoyo del tratamiento oncológico. Su principal efecto pro hipertensivo se ejerce a través de la acción mineralo-corticoide que induce retención hidrosalina, aunque también incrementan la síntesis de angiotensinógeno. Al menos $20 \%$ de los pacientes que reciben corticoterapia prolongada tienen elevación tensional significativa y este efecto es dependiente de la dosis utilizada, siendo más marcado en pacientes de edad avanzada, con HTA previa o disfunción renal. El tratamiento con diuréticos e IECA contrarresta el efecto presor de estas drogas ${ }^{(27)}$.

\section{Manejo de la hipertensión arterial en el paciente con cáncer}

No existen guías basadas en evidencia firme para orientar el tratamiento de la HTA en el paciente con cáncer y las principales normativas sobre HTA (procedentes del American College of Cardiology/American Heart Association y European Society of Cardiology/European Society of Hypertension) apenas mencionan el tema ${ }^{(28,29)}$. De modo que si bien los lineamientos generales que establecen las mencionadas guías sobre diagnóstico y tratamiento tienen plena vigencia en los pacientes con cáncer, en aspectos más específicos del manejo debemos basarnos en opiniones de expertos. En el año 2010 se publicó un consenso auspiciado por el Instituto Nacional del Cáncer de Estados Unidos sobre el manejo de la PA entre los pacientes que reciben agentes IA, que fueron reafirmados en un segundo documento en $2012^{(22,30)}$. En 2016, la Sociedad Europea de Cardiología emitió un documento sobre toxicidad cardiovascular durante el tratamiento del cáncer que dedica un capítulo al manejo de la HTA $^{(21)}$. En nuestra región las sociedades argentina y brasileña de cardiología también han emitido recomendaciones al respecto ${ }^{(12,13,31)}$.

Si bien estos documentos se dedican preferentemente a las drogas IA, las recomendaciones genera- 
Tabla 2. Precauciones, contraindicaciones y motivos de preferencia para las clases mayores de drogas antihipertensivas durante tratamiento oncológico. Adaptado de Maitland et al, referencia 30.

\begin{tabular}{lll} 
Clase de droga & Precauciones en relación al cáncer & \multicolumn{1}{c}{ Motivos de preferencia } \\
\hline $\begin{array}{l}\text { Inhibidores de enzima } \\
\text { conversora de angiotensina } \\
\text { (IECA) }\end{array}$ & $\begin{array}{l}\text { Precaución de uso junto con Disfunción sistólica del } \\
\text { drogas que dependen de ventrículo izquierdo, } \\
\text { función renal (cisplatino, nefropatía diabética } \\
\text { pemetrexed); hiperkalemia }\end{array}$ \\
$\begin{array}{l}\text { Antagonistas de } \\
\text { angiotensina II (ARA II) }\end{array}$ & $\begin{array}{l}\text { Precaución de uso junto con Intolerancia a los IECA. } \\
\text { drogas que dependen de } \\
\text { función renal (cisplatino, Disfunción sistólica del } \\
\text { pemetrexed); hiperkalemia nefropatía diabética. }\end{array}$
\end{tabular}

Betabloqueantes

fatiga; drogas que
prolongan el intervalo QT

Antagonistas cálcicos (dihidropiridínicos)

Diuréticos tiazídicos

\section{Edema de miembros} inferiores
Ángor; historia de infarto de miocardio; disfunción ventricular izquierda; ansiedad

Pacientes ancianos; hipertensión sistólica aislada

Pacientes ancianos; hipertensión sistólica

Gota; hipercalcemia hipokalemia; edad $<45$ años; drogas que prolongan el QT reducido
Precauciones y contraindicaciones

Enfermedad reno-vascular, enfermedad vascular periférica, disfunción renal severa.

Enfermedad reno-vascular; enfermedad vascular periférica; disfunción renal severa.

Bradiarritmia; diabetes (hipoglicemia); broncoespasmo; falla cardíaca descompensada

Edema preexistente; lento inicio de acción

Gota; alergia documentada a sulfas; hipokalemia; deshidratación

* Diltiazem y verapamil interfieren metabolismo hepático de sorafenib y sunitinib (CYP3A4); se debería evitar uso conjunto.

les son aplicables al manejo de la HTA en todos los pacientes que reciben tratamiento oncológico.

\section{Recomendaciones básicas}

1. Es necesario realizar una evaluación formal del riesgo cardiovascular en todos los pacientes que recibirán tratamiento por cáncer, pues es la más frecuente comorbilidad y con frecuencia condiciona el manejo oncológico. La HTA preexistente debe ser identificada y tratada desde el comienzo.

2. Se recomienda un monitoreo activo de la PA en los pacientes que reciben quimioterapia, especialmente si reciben alguna de las drogas que pueden inducir HTA. Cuando se utilizan agentes IA, el monitoreo debe ser más cuidadoso en el primer ciclo de tratamiento, pues allí se presentan la mayoría de los casos con incremento inesperado y a veces severo de la PA, aun en pacientes sin factores de riesgo.

3. Los objetivos de PA a obtener durante el tratamiento deben seguir las directivas generales del manejo de la HTA establecidas por las diferentes sociedades científicas. Si bien estas guías han tenido numerosas variaciones en los últimos años, se mantiene vigente un objetivo menor de 140/90 para todos los pacientes, pudiendo contemplar objetivos menores $(<130 / 80)$ en casos seleccionados por su alto riesgo.
4. El manejo de la HTA debe ser inmediato y agresivo para evitar a la vez repercusión sobre órganos blanco y dificultades en el tratamiento del cáncer que obliguen a modificar o suspender el mismo.

5. Salvo la restricción de sodio, las medidas no farmacológicas tienen un espacio muy limitado en el contexto del tratamiento del cáncer avanzado, por las condiciones que impone en lo nutricional y en la capacidad de realizar ejercicio físico.

\section{Elección de drogas antihipertensivas}

Todos los tipos de drogas antihipertensivas de primera línea (diuréticos tiazídicos, IECA, ARA II, calcioantagonistas y betabloqueantes) han sido utilizados durante el tratamiento del cáncer y todos se han mostrado razonablemente eficaces. Aquí rige el principio general del tratamiento antihipertensivo: es más importante la obtención del objetivo en cifras tensionales que el tipo de droga utilizada para ello.

En la tabla 2 se desarrollan los principales aspectos a considerar cuando se elige un agente antihipertensivo, tomando en cuenta precauciones o contraindicaciones relativas al cáncer o su tratamiento, junto con indicaciones obligatorias o precauciones que surgen del contexto médico general de cada paciente. 
Dado que el principal efecto pro hipertensivo propuesto para las drogas IA es mediado por la inhibición de la vía del óxido nítrico, las drogas que aumentan la disponibilidad del mismo, como los IECA o el betabloqueante nevibolol, pueden ser una elección razonable. Asimismo, se ha propuesto el carvedilol, un betabloqueante con acción vasodilatadora, como una alternativa válida ${ }^{(21,27,32)}$.

El verapamil y el diltiazem inhiben la vía del citocromo P450 (CYP3A4) e interfieren por este mecanismo con el metabolismo hepático de sunitinib, sorafenib y otras drogas similares, por lo que no deberían utilizarse en conjunto ${ }^{(27,30)}$.

El uso de diuréticos debe ser cauteloso por la posibilidad de generar o agravar trastornos hidroelectrolíticos, dado que las drogas IA pueden producir diarrea y deshidratación.

Muchos expertos opinan que una combinación en dosis bajas de un IECA (o un ARA II) y amlodipina es una elección eficaz para controlar la HTA inducida por agentes antiangiogénicos. Cuando apremia un rápido comienzo de efecto y breve período de titulación de la dosis, un IECA suele ofrecer la respuesta más adecuada como tratamiento inicial ${ }^{(27,30)}$.

\section{Modificaciones del tratamiento oncológico}

$\mathrm{Al}$ inicio del tratamiento con drogas IA es conveniente asegurarse que la PA es normal o está bien controlada. De otro modo el tratamiento con IA debería posponerse algunos días hasta conseguir un buen control.

El efecto hipertensivo de estos agentes es reversible, por lo que una disminución de la dosis o la interrupción temporaria del tratamiento pueden ser utilizadas, como último recurso, para controlar un brote severo de HTA. Esto se aplica sobre todo a los inhibidores de tirosina quinasa, pues los mismos se administran diariamente por vía oral por períodos prolongados y pueden reiniciarse, o ajustar la dosis, una vez que la HTA se ha controlado con la medicación adecuada.

Cuando se utiliza bevacizumab, $\mathrm{u}$ otros agentes de administración endovenosa y efecto prolongado, no puede esperarse una rápida disminución del efecto hipertensivo después de inyectados y una reducción de dosis tiene efecto desconocido. Usualmente se recomienda no administrar bevacizumab si la presión sistólica es mayor de $160 \mathrm{mmHg}$ o la diastólica mayor de $100 \mathrm{mmHg}$, o frente a cualquier nivel de hipertensión sintomática al momento del tratamiento. Una vez controlada la situación con el tratamiento antihipertensivo durante el período de suspensión transitoria (usualmente cuatro semanas), la droga puede volver a ser administrada ${ }^{(12,30)}$.
En pacientes que finalizan el tratamiento con un agente IA es esperable que la PA vuelva lentamente a sus niveles previos, por lo que pueden necesitar reducir la dosis o interrumpir el tratamiento antihipertensivo.

\section{Bibliografía}

1. Organización Mundial de la Salud. Información general sobre la hipertensión en el mundo: una enfermedad que mata en silencio, una crisis de salud pública mundial. Ginebra: OMS, 2013. Disponible en: http://www.who.int/cardiovascular_diseases/publications/global_brief_hypertension/es/. [Consulta:28/01/ 2018].

2. Piccirillo JF, Tierney RM, Costas I, Grove L, Spitznagel EL Jr. Prognostic importance of comorbidity in a hospital-based cancer registry. JAMA 2004; 291(20):2441-7.

3. Smith AW, Reeve BB, Bellizzi KM, Harlan LC, Klabunde CN, Amsellem M, et al. Cancer, comorbidities, and health-related quality of life of older adults. Health Care Financ Rev 2008; 29(4):41-56.

4. Mouhayar E, Salahudeen A. Hypertension in cancer patients. Tex Heart Inst J 2011; 38(3):263-5.

5. Stocks T, Van Hemelrijck M, Manjer J, Bjørge T, Ulmer H, Hallmans G, et al. Blood pressure and risk of cancer incidence and mortality in the Metabolic Syndrome and Cancer Project. Hypertension 2012; 59(4):802-10.

6. Han H, Guo W, Shi W, Yu Y, Zhang Y, Ye X, et al. Hypertension and breast cancer risk: a systematic review and meta-analysis. Sci Rep 2017; 7:44877.

7. Fraeman KH, Nordstrom BL, Luo W, Landis SH, Shantakumar S. Incidence of new-onset hypertension in cancer patients: a retrospective cohort study. Int J Hypertens 2013; 2013:379252.

8. Hershman DL, McBride RB, Eisenberger A, Tsai WY, Grann VR, Jacobson JS. Doxorubicin, cardiac risk factors, and cardiac toxicity in elderly patients with diffuse B-cell non-Hodgkin's lymphoma. J Clin Oncol 2008; 26(19):3159-65.

9. Chu TF, Rupnick MA, Kerkela R, Dallabrida SM, Zurakowski D, Nguyen L, et al. Cardiotoxicity associated with tyrosine kinase inhibitor sunitinib. Lancet 2007; 370(9604):2011-9.

10. Yeh ET, Bickford CL. Cardiovascular complications of cancer therapy: incidence, pathogenesis, diagnosis, and management. J Am Coll Cardiol 2009; 53(24):2231-47.

11. Meinardi MT, Gietema JA, van der Graaf WT, van Veldhuisen DJ, Runne MA, Sluiter WJ, et al. Cardiovascular morbidity in long-term survivors of metastatic testicular cancer. J Clin Oncol 2000; 18(8):1725-32. 
12. Sociedad Argentina de Cardiología. Consenso sobre diagnóstico, prevención y tratamiento de la cardiotoxicidad por tratamiento médico del cáncer. Rev Argent Cardiol 2013; 81(Supl 5):1-64

13. Souza V Barbosa de, Silva E, Ribeiro ML, Andrade M Wolney de. Hipertensão arterial no paciente com câncer. Arq Bras Cardiol 2015 104(3):246-52.

14. Vasudev NS, Reynolds AR. Anti-angiogenic therapy for cancer: current progress, unresolved questions and future directions. Angiogenesis 2014 17(3):471-94.

15. Kamba T, McDonald DM. Mechanisms of adverse effects of anti-VEGF therapy for cancer. Br J Cancer 2007; 96(12):1788-95.

16. Izzedine H, Ederhy S, Goldwasser F, Soria JC, Milano G, Cohen A, et al. Management of hypertension in angiogenesis inhibitor-treated patients. Ann Oncol 2009; 20(5):807-15.

17. Österlund P, Soveri LM, Isoniemi H, Poussa T, Alanko T, Bono P. Hypertension and overall survival in metastatic colorectal cancer patients treated with bevacizumab-containing chemotherapy. $\mathrm{Br} \mathrm{J}$ Cancer 2011; 104(4):599-604.

18. Wasserstrum Y, Kornowski R, Raanani P, Lea$\operatorname{der}$ A, Pasvolsky O, Iakobishvii Z. Hypertension in cancer patients trated with anti-angiogenic based regimens. Cardio-Oncol 2015; 1:6.

19. Rini BI, Cohen DP, Lu DR, Chen I, Hariharan S, Gore ME, et al. Hypertension as a biomarker of efficacy in patients with metastatic renal cell carcinoma treated with sunitinib. J Natl Cancer Inst 2011; 103(9):763-73.

20. Syrigos KN, Karapanagiotou E, Boura P, Manegold $\mathbf{C}$, Harrington $\mathrm{K}$. Bevacizumab-induced hypertension: pathogenesis and management. BioDrugs 2011; 25(3):159-69.

21. Zamorano JL, Lancellotti P, Rodriguez Muñoz D, Aboyans V, Asteggiano R, Galderisi M, et al. 2016 ESC position paper on cancer treatments and cardiovascular toxicity developed under the auspices of the ESC Committee for Practice Guidelines: ?The Task Force for cancer treatments and cardiovascular toxicity of the European Society of Cardiology (ESC). Eur Heart J 2016; 37(36):2768-2801.

22. Steingart RM, Bakris GL, Chen HX, Chen MH, Force T, Ivy SP, et al. Management of cardiac toxicity in patients receiving vascular endothelial growth factor signaling pathway inhibitors. Am Heart $\mathrm{J}$ 2012; 163(2):156-63.

23. Ranpura V, Pulipati B, Chu D, Zhu X, Wu S. Increased risk of high-grade hypertension with beva- cizumab in cancer patients: a meta-analysis. Am J Hypertens 2010; 23(5):460-8.

24. Hamnvik OP, Choueiri TK, Turchin A, McKay RR, Goyal L, Davis M, et al. Clinical risk factors for the development of hypertension in patients treated with inhibitors of the VEGF signaling pathway. Cancer 2015; 121(2):311-9.

25. Maitland ML, Kasza KE, Karrison T, Moshier K, Sit L, Black HR, et al. Ambulatory monitoring detects sorafenib-induced blood pressure elevations on the first day of treatment. Clin Cancer Res 2009; 15(19):6250-7.

26. Azizi M, Chedid A, Oudard S. Home blood-pressure monitoring in patients receiving sunitinib. $\mathrm{N}$ Engl J Med 2008; 358(1):95-7.

27. Rossi GP, Seccia TM, Maniero C, Pessina AC. Drug-related hypertension and resistance to antihypertensive treatment: a call for action. J Hypertens 2011; 29(12):2295-309.

28. Mancia G, Fagard R, Narkiewicz K, Redon J, Zanchetti A, Böhm M, et al. 2013 ESH/ESC guidelines for the management of arterial hypertension: the Task Force for the Management of Arterial Hypertension of the European Society of Hypertension (ESH) and of the European Society of Cardiology (ESC). Eur Heart J 2013; 34(28):2159-219.

29. Whelton PK, Carey RM, Aronow WS, Casey DE Jr, Collins KJ, Dennison Himmelfarb C, et al. 2017 ACC/AHA/AAPA/ABC/ACPM/AGS/APhA/ ASH/ASPC/NMA/PCNA Guideline for the prevention, detection, evaluation, and management of high blood pressure in adults: executive summary. A report of the American College of Cardiology/American Heart Association Task Force on Clinical Practice Guidelines. J Am Coll Cardiol 2017; pii: S0735-1097(17)41518-X. doi: 10.1016/j.jacc.2017.11.005. [Epub ahead of print].

30. Maitland ML, Bakris GL, Black HR, Chen HX, Durand JB, Elliott WJ, et al. Initial assessment, surveillance, and management of blood pressure in patients receiving vascular endothelial growth factor signaling pathway inhibitors. J Natl Cancer Inst 2010; 102(9):596-604.

31. Kalil Filho R, Hajjar LA, Bacal F, Hoff PM, Diz MD, Galas RB, et al. I Diretriz Brasileira de cardio-oncologia da Sociedade Brasileira de Cardiologia. Arq Bras Cardiol 2011; $96(2$ supl 1):1-52.

32. Hall PS, Harshman LC, Srinivas S, Witteles RM. The frequency and severity of cardiovascular toxicity from targeted therapy in advanced renal cell carcinoma patients. JACC Heart Fail 2013; $1(1): 72-8$. 